\title{
Two-Pressure Model of Particle-Fluid Mixture Flow with Pressure-Dependent Viscosity in a Porous Medium
}

\author{
S. JAYYOUSI DAJANI \\ Department of Mathematics and Computer Science \\ Lake Forest College \\ Lake Forest, IL 60045 \\ USA \\ M.S. ABU ZAYTOON \\ Department of Mathematics \\ University of Petra, Amman \\ JORDAN \\ M.H. HAMDAN \\ Department of Mathematics and Statistics \\ University of New Brunswick \\ Saint John, N.B., E2L 4L5 \\ CANADA
}

\begin{abstract}
Equations governing the flow of a fluid-particle mixture with variable viscosity through a porous structure are developed. Method of intrinsic volume averaging is used to average Saffman's dusty gas equations. A modelling flexibility is offered in this work by introducing a dust-phase partial pressure in the governing equations, interpreted as the pressure necessary to maintain a uniform particle distribution in the flow field. Viscosity of the fluid-particle mixture is assumed to be variable, with variations in viscosity being due to fluid pressure. Particles are assumed spherical and Stokes' coefficient of resistance is expressed in terms of the pressure-dependent fluid viscosity. Both Darcy resistance and the Forchheimer micro-inertial effects are accounted for in the developed model.
\end{abstract}

Key-Words: - Pressure-dependent viscosity, fluid-particle mixture, porous media.

Received: February 10, 2021. Revised: August 2, 2021. Accepted: August 13, 2021. Published: August 17, 2021.

\section{Introduction}

The past two decades have witnessed increasing research activity in the field of flow through porous media of fluids with pressure-dependent viscosities. This may be ascribed to the many applications found in modern industry and involve fluids with variable viscosity, such as the oil industry (enhanced oil recovery and carbon sequestration), lubrication theory (where lubricants experience extreme pressures that change their viscosities), and in natural phenomena that involves filtration problems in earth layers. For these and many other applications, one is referred to the works reported in [1 ], [2], [3], and the references therein.

A large portion of research work on flow of pressure-dependent viscosity revolves around the modelling of the flow phenomena. This is due to the fact that the available models of flow through porous media have largely been centred on flows with constant viscosity and there is a need for new models that take into account variations in viscosity, especially due to high pressure gradients. This in turn resulted in new paradigms of generalized versions of the available models. A number of models are 
available and can be found in the works of Chang et.al. [4], Abu Zaytoon et.al. [5], Alharbi et.al. [6].

While the above models are gaining popularity, they only describe flows of a single-phase pressuredependent viscosity fluid through porous structures. Models representing the flow of a gas-particle mixture with pressure-dependent viscosity are still at their infancy, although some models have been developed over the past five years, [7,8]. Abu Zaytoon and Hamdan [7] developed a pressuredependent viscosity dusty gas model of flow through porous media with variable number density based on Saffman's dusty gas model [9]. Roach et.al. [8] developed a pressure-dependent viscosity dusty gas model of flow based on Marble's equations, [10].

Modelling gas-particle mixture flow through porous structures based on the continuum approach and the intrinsic volume averaging of Saffman's dusty gas model, [9], was first introduced by Barron and Hamdan, [11, 112], more than three decades ago. Various other models taking into account the porous microstructure have also been developed with the view of applicability in filtration processes and the design of liquid-dust separators, (cf. $[13,14]$ and the references therein).

In modelling fluid-particle mixtures in which the carrier fluid possesses a pressure-dependent viscosity, models available to date are one-pressure models in which the fluid pressure is the only pressure, and the flow is driven by a fluid pressure gradient. An alternative is to model this special twophase flow as a two-pressure system, wherein a dustphase partial pressure is introduced. This is the subject matter of this work, and has the advantage of offering the modelling flexibility of describing flow of a dusty gas with uniform particle distribution. The dust-phase partial pressure may be interpreted in this case as the pressure needed to maintain a uniform dust particle distribution in the flow field. Clearly, this offers a modelling advantage and a flexibility in which one model can serve both unifom particle distribution (whenein the dust-phase partial pressure is non-zero) and the general non-uniform particle distribution (in which case we take the dust-phase partial pressure as zero).

In order to accomplish this work, we provide intrinsic volume averaging of Saffman's dusty gas model with a fluid-pressure and a dust-phase partial pressure. The effects of the porous microstructure on the flowing phases is considered and frictional forces involving Darcy's viscous drag and Forchheimer's inertial drag terms are included. Dependence of viscosity on pressure is accounted for through Barus' relationships, $[15,16]$.

\section{Model Development}

\subsection{Governing Equations in Free Space}

Consider a rigid porous medium through which an incompressible dusty gas with a small concentration of dust particles per unit volume flows under the action of a pressure gradient. The flow is assumed to be steady and the dust particles spherical. In free space, the flow is governed by the following set of coupled field equations developed by Saffman, [9]:

Fluid-phase continuity equation

$\boldsymbol{\nabla} \cdot \overrightarrow{\boldsymbol{u}}=0$

Fluid-phase momentum equation

$\rho \boldsymbol{\nabla} \cdot \overrightarrow{\boldsymbol{u} u}=-\nabla p+\boldsymbol{\nabla} \cdot \overrightarrow{\boldsymbol{T}}+\frac{9}{2 a^{2}} \mu N(\overrightarrow{\boldsymbol{v}}-\overrightarrow{\boldsymbol{u}})$

Dust-phase continuity equation

$\boldsymbol{\nabla} \cdot N \overrightarrow{\boldsymbol{v}}=0$

Dust-phase momentum equation

$m \boldsymbol{\nabla} \cdot N \overrightarrow{\boldsymbol{v}} \boldsymbol{v}=\frac{9}{2 a^{2}} \mu N(\overrightarrow{\boldsymbol{u}}-\overrightarrow{\boldsymbol{v}})$

wherein

$\overrightarrow{\boldsymbol{T}}=\mu\left(\boldsymbol{\nabla} \overrightarrow{\boldsymbol{u}}+\boldsymbol{\nabla} \overrightarrow{\boldsymbol{u}}^{T}\right)$

and $\overrightarrow{\boldsymbol{u}}$ and $\overrightarrow{\boldsymbol{v}}$ are the fluid-phase and dust-phase velocity fields, respectively, $p$ is the fluid pressure, $\rho$ is the constant fluid density, $m$ is the mass of a dust particle, $N$ is the particle distribution (or the particle number density, that is, the number of particles per unit volume), $a$ is the spherical particle diameter and $\mu$ is the fluid viscosity coefficient. The term $\frac{9 \mu}{2 a^{2}}$ is the Stokes' coefficient of resistance that is valid for spherical particles. Clearly, if the viscosity is variable then the Stokes' coefficient of resistance varies locally.

Equations (1) through (4) represent a determinate system of eight scalar equations in the eight unknowns, $\overrightarrow{\boldsymbol{u}}, \overrightarrow{\boldsymbol{v}}, N$ and $p$ as functions of position, $\overrightarrow{\boldsymbol{x}}$. We point out that the form of equations (3) and (4) can be used for both variable and constant number density, $N$. If $N$ is constant, the dust particle 
distribution is said to be uniform, and equations (3) and (4) can be written, respectively, as:

$\boldsymbol{\nabla} \cdot \overrightarrow{\boldsymbol{v}}=0$

$m \boldsymbol{\nabla} \cdot \overrightarrow{\boldsymbol{v}} \boldsymbol{v}=\frac{9}{2 a^{2}} \mu(\overrightarrow{\boldsymbol{u}}-\overrightarrow{\boldsymbol{v}})$

A uniform particle distribution implies that the number density $N$ is constant along the dust-phase streamlines. This can be seen by writing (3) as:

$\boldsymbol{\nabla} \cdot N \overrightarrow{\boldsymbol{v}}=N \boldsymbol{\nabla} \cdot \overrightarrow{\boldsymbol{v}}+\overrightarrow{\boldsymbol{v}} \cdot \boldsymbol{\nabla} N=0$

Using (6) in (8), we obtain

$\overrightarrow{\boldsymbol{v}} \cdot \nabla N=0$

Equation (9) implies that $N$ is constant along the dust-phase streamlines.

For a dusty gas flow with uniform particle distribution, the governing equations are (1), (2), (6), and (7). These represent an over-determined system of eight scalar equations in seven unknowns. One way to generate a determinate system is to introduce a dust-phase partial pressure, $p_{d}$, and write equation (7) in the following form:

$$
m \boldsymbol{\nabla} \cdot N \overrightarrow{\boldsymbol{v}} \overrightarrow{\boldsymbol{v}}=-N \nabla p_{d}+\frac{9}{2 a^{2}} \mu N(\overrightarrow{\boldsymbol{u}}-\overrightarrow{\boldsymbol{v}})
$$

The dust-phase partial pressure may be interpreted as the force necessary to be applied to maintain a uniform distribution of dust particles in the flow domain. Our governing equations are thus (1), (2), (7) and (10). They represent a determinate system of eight scalar equations in the eight unknowns $\overrightarrow{\boldsymbol{u}}, \overrightarrow{\boldsymbol{v}}, p_{d}$ and $p$, and are valid for a uniform particle distribution.

The introduction of the dust-phase partial pressure allows us some modeling flexibility, as follows: If the dust-phase partial pressure is taken as zero in equation (10), we recover the original momentum equation (4). With $N$ being a variable, equations (1), (2), (3), (4) represent a determinate system of eight scalar equations in the eight unknowns $\overrightarrow{\boldsymbol{u}}, \overrightarrow{\boldsymbol{v}}, N$ and $p$. If the dust particle distribution is uniform, $N$ is taken as constant and the dust-phase partial pressure is non-zero in equation (10). Equations (1), (2), (7), and (10) represent a determinate system of eight scalar equations in the eight unknowns $\overrightarrow{\boldsymbol{u}}, \overrightarrow{\boldsymbol{v}}, p_{d}$ and $p$.

\subsection{Governing Equations in a Porous Structure}

In order to develop a continuum model to describe the flow of a particle-fluid mixture with either a uniform or a non-uniform particle distribution through an isotropic porous material of variable porosity, we follow the method of intrinsic volume averaging. Governing equations (1), (2), (3) and (10) will be averaged over a Representative Elementary Volume $(R E V)$, which is a control volume that embeds within it properties of the porous medium. Letting $V$ be the bulk volume of the REV and $V_{\varphi}$ its pore volume, porosity of the REV (and that of the porous medium) is given by $\varphi=\frac{V_{\varphi}}{V}$.

We will make the further assumption that fluid viscosity is variable. This renders the system of governing equations (1), (2), (3) and (10) an underdetermined system of eight equations in the nine unknowns $\overrightarrow{\boldsymbol{u}}, \overrightarrow{\boldsymbol{v}}, p_{d}, p$ and $\mu$. To remedy, we take the viscosity as a known function of pressure, $\mu=$ $\mu(p)$.

Following Du Plessis and Masliyah [17, 18], the following notation and rules for volume averaging are listed here for ease of reference.

The volumetric phase average of a quantity $F$ (that is, the volumetric volume average of $F$ over the bulk volume, $V$ ) is defined as:

$$
<F>=\frac{1}{V} \iiint_{V_{\varphi}} F d V \equiv \frac{1}{V} \int_{V_{\varphi}} F d V
$$

and the intrinsic phase average (that is, the volumetric average of $F$ over the effective pore space, $V_{\phi}$ ) is defined as:

$$
<F>_{\varphi}=\frac{1}{V_{\varphi}} \int_{V_{\varphi}} F d V
$$

Relationship between the volumetric phase average and the intrinsic phase average is obtained from equations (i), (ii) and the definition of porosity, $\varphi=\frac{V_{\varphi}}{V}$, and takes the form:

$$
<F>=\varphi<F>_{\varphi}
$$

Averaging theorems are written in the following forms. Let $F$ and $H$ be volumetrically additive scalar quantities, $\vec{F}$ a vector quantity, and $c$ a constant, then:

(iv) $\quad\langle c\rangle=c$

(v) $\quad\left\langle c F>=c<F>=c \varphi<F>_{\varphi}\right.$ 


$$
<\boldsymbol{\nabla} F>=\varphi \boldsymbol{\nabla}<F>_{\varphi}+\frac{1}{V} \int_{S} F^{\circ} \overrightarrow{\boldsymbol{n}} d S
$$

where $S$ is the surface area of the solid matrix in the REV that is in contact with the fluid, and $\overrightarrow{\boldsymbol{n}}$ is the unit normal vector pointing into the solid. The quantity $F^{\circ}=F-<F>=F-\varphi<F>_{\varphi}$ is the deviation of the averaged quantity from its true (microscopic) value.

$$
\begin{array}{ll}
\text { (vii) } \quad & <F \mp H>=<F>\mp<H>=\varphi< \\
& F>_{\varphi} \mp \varphi<H>_{\varphi}=\varphi\left(<F>_{\varphi} \mp<\right. \\
& \left.H>{ }_{\varphi}\right) \\
\text { (viii) } \quad<F H>=\varphi<F H>_{\varphi}=\varphi<F>_{\varphi}< \\
\\
\quad H>_{\varphi}+\varphi<F^{\circ} H^{\circ}>_{\varphi}
\end{array}
$$

$$
\text { (ix) } \quad\left\langle\boldsymbol{\nabla} \cdot \overrightarrow{\boldsymbol{F}}>=\boldsymbol{\nabla} \cdot \varphi<\overrightarrow{\boldsymbol{F}}>_{\varphi}+\right.
$$
$\frac{1}{V} \int_{S} \overrightarrow{\boldsymbol{F}} \cdot \overrightarrow{\boldsymbol{n}} d S$

(x) Due to the no-slip condition, a surface integral is zero if it contains the fluid velocity vector explicitly.

The above averaging rules are applied to equations (1), (2), (3) and (10) to obtain the following forms.

\section{For fluid-phase:}

\section{Continuity Equation:}

Taking the averages of both sides of (1), and invoking rules (iv) and (viii), we obtain

$\boldsymbol{\nabla} \cdot \varphi<\overrightarrow{\boldsymbol{u}}>_{\varphi}+\frac{1}{V} \int_{S} \overrightarrow{\boldsymbol{u}} \cdot \overrightarrow{\boldsymbol{n}} d S=0$

\section{Momentum Equations:}

Taking the averages of both sides of (2), and invoking rules (v) and (ix), we obtain

$$
\begin{aligned}
& \rho \nabla \cdot \varphi<\overrightarrow{\boldsymbol{u}}>_{\varphi}<\overrightarrow{\boldsymbol{u}}>_{\varphi}+\rho \nabla \cdot \varphi<\overrightarrow{\boldsymbol{u}}^{\circ} \overrightarrow{\boldsymbol{u}}^{\circ}>_{\varphi}+ \\
& \frac{\rho}{V} \int_{S} \overrightarrow{\boldsymbol{u}} \overrightarrow{\boldsymbol{u}} \cdot \overrightarrow{\boldsymbol{n}} d S=-\varphi \nabla<p>_{\varphi}-\frac{1}{V} \int_{S} p^{\circ} \overrightarrow{\boldsymbol{n}} d S+\nabla \cdot \\
& \varphi<\overrightarrow{\boldsymbol{T}}>_{\varphi}+\frac{1}{V} \int_{S} \overrightarrow{\boldsymbol{T}} \cdot \overrightarrow{\boldsymbol{n}} d S+\frac{9}{2 a^{2}} \varphi<\mu>_{\varphi}< \\
& N>_{\varphi}\left[\overrightarrow{\boldsymbol{v}}>_{\varphi}-<\overrightarrow{\boldsymbol{u}}>_{\varphi}\right]+\frac{9}{2 a^{2}} \varphi\left(<\mu^{\circ} N^{\circ} \overrightarrow{\boldsymbol{v}}^{\circ}>_{\varphi}-<\right. \\
& \left.\mu^{\circ} N^{\circ} \overrightarrow{\boldsymbol{u}}^{\circ}>_{\varphi}\right)
\end{aligned}
$$

\section{For dust-phase:}

\section{Continuity Equation:}

Upon taking the averages of both sides of equation (3), and invoking rules (iv), (vii), (viii) and (ix), we obtain

$$
\begin{aligned}
& \boldsymbol{\nabla} \cdot \varphi<N>_{\varphi}<\overrightarrow{\boldsymbol{v}}>_{\varphi}+\boldsymbol{\nabla} \cdot \varphi<N^{\circ} \overrightarrow{\boldsymbol{v}}^{\circ}>_{\varphi}+ \\
& \frac{1}{V} \int_{S} N \overrightarrow{\boldsymbol{v}} \cdot \overrightarrow{\boldsymbol{n}} d S=0
\end{aligned}
$$

\section{Momentum Equations:}

Taking the averages of both sides of equation (10), and invoking rules (v), (vi), (vii), (viii) and (ix), we obtain

$$
\begin{gathered}
m \boldsymbol{\nabla} \cdot \varphi<N>_{\varphi}<\overrightarrow{\boldsymbol{v}}>_{\varphi}<\overrightarrow{\boldsymbol{v}}>_{\varphi}+m \boldsymbol{\nabla} \cdot \varphi \\
<N^{\circ} \overrightarrow{\boldsymbol{v}}^{\circ} \overrightarrow{\boldsymbol{v}}^{\circ}>_{\varphi}+\frac{m}{V} \int_{S} N \overrightarrow{\boldsymbol{v}} \overrightarrow{\boldsymbol{v}} \cdot \overrightarrow{\boldsymbol{n}} d S \\
=-\varphi<N>_{\varphi} \nabla<p_{d}>_{\varphi}-\varphi<N^{\circ}\left(\nabla p_{d}\right)^{\circ}>_{\varphi}- \\
\frac{N}{V} \int_{S} p_{d}^{\circ} \overrightarrow{\boldsymbol{n}} d S+\frac{9}{2 a^{2}}<\mu>_{\varphi}<N>_{\varphi}\left(<\overrightarrow{\boldsymbol{u}}>_{\varphi}-<\right. \\
\left.\overrightarrow{\boldsymbol{v}}>_{\varphi}\right)+\frac{9}{2 a^{2}} \varphi\left(<\mu^{\circ} N^{\circ} \overrightarrow{\boldsymbol{u}}^{\circ}>_{\varphi}-<\mu^{\circ} N^{\circ} \overrightarrow{\boldsymbol{v}}^{\circ}>_{\varphi}\right)(14)
\end{gathered}
$$

\section{Analysis of Surface Integrals and Deviation Terms}

Equations (11), (12), (13) and (14) represent the intrinsic volume averages of equations (1), (2), (3) and (10), respectively. The deviation terms and surface integrals that appear in these averaged equations contain information on the forces that are exerted by the porous matrix on the fluid-phase and dust phase, and the interactions that take place between the phases involved. Pore space boundaries present additional solid boundary on which the fluidphase experiences no-slip on its velocity and the dustphase experiences additional friction that results in dust particle capture mechanisms, settling, and reflection into the flow field. Tortuosity of the flow path and the converging-diverging pore structure could result in enhancing microscopic inertial effects or may influence dispersion of the dust particles. It is therefore important to accurately analyze the above surface integrals and deviation terms.

\subsection{Analysis of the Deviation Terms}

Deviations from microscopic quantities are present in the fluid-phase momentum equations and in the dustphase continuity and momentum equations. Products of deviations can be identified with hydrodynamic dispersion of the average phase velocities in the porous medium, [17, 18]. Hydrodynamic dispersion through porous media is the sum of mechanical dispersion due to tortuosity of the flow path in the 
porous microstructure, and molecular diffusion of vorticity.

The inertial deviation terms, $\left.\boldsymbol{\nabla} \cdot \varphi<\overrightarrow{\boldsymbol{u}}^{\circ} \overrightarrow{\boldsymbol{u}}^{\circ}\right\rangle_{\varphi}$ of equation (12) and $\boldsymbol{\nabla} \cdot \varphi<N^{\circ} \overrightarrow{\boldsymbol{v}}^{\circ} \overrightarrow{\boldsymbol{v}}^{\circ}>_{\varphi}$ of equation (14), involve averages of products of deviations of average phase velocities and are representative of mechanical dispersion due to the porous microstructure. Using rule (viii), we can write:

$$
\begin{aligned}
& <\overrightarrow{\boldsymbol{u}}^{\circ} \overrightarrow{\boldsymbol{u}}^{\circ}>_{\varphi}=<\overrightarrow{\boldsymbol{u}}^{\circ}>_{\varphi}<\overrightarrow{\boldsymbol{u}}^{\circ}>_{\varphi}+<\overrightarrow{\boldsymbol{u}}^{\circ \circ} \overrightarrow{\boldsymbol{u}}^{\circ \circ}>_{\varphi} \\
& <N^{\circ} \overrightarrow{\boldsymbol{v}}^{\circ} \overrightarrow{\boldsymbol{v}}^{\circ}>_{\varphi}=<N^{\circ}>_{\varphi}<\overrightarrow{\boldsymbol{v}}^{\circ}>_{\varphi}<\overrightarrow{\boldsymbol{v}}^{\circ}>_{\varphi}+< \\
& N^{\circ \circ} \overrightarrow{\boldsymbol{v}}^{\circ \circ} \overrightarrow{\boldsymbol{v}}^{\circ \circ}>_{\varphi}
\end{aligned}
$$

The leading terms on the right-hand-sides of (15) and (16) involve products of averages of deviations that are arguably small in porous media where velocity and porosity gradients are not high, hence can be neglected. However, they may be of significance in media with high porosity gradients, hence a need arises to model them using dynamic diffusivity, $[13,14]$.

The term $<\mu^{\circ} N^{\circ} \overrightarrow{\boldsymbol{v}}^{\circ}>_{\varphi}-<\mu^{\circ} N^{\circ} \overrightarrow{\boldsymbol{u}}^{\circ}>_{\varphi}$ in (12) and its negative in (14), can be written as:

$$
\begin{aligned}
& <\mu^{\circ} N^{\circ} \overrightarrow{\boldsymbol{v}}^{\circ}>_{\varphi}-<\mu^{\circ} N^{\circ} \overrightarrow{\boldsymbol{u}}^{\circ}>_{\varphi}=<\mu^{\circ}>_{\varphi}< \\
& N^{\circ}>_{\varphi}\left(<\overrightarrow{\boldsymbol{v}}^{\circ}>_{\varphi}-<\overrightarrow{\boldsymbol{u}}^{\circ}>_{\varphi}\right)+(< \\
& \mu^{\circ \circ} N^{\circ \circ} \overrightarrow{\boldsymbol{v}}^{\circ \circ}>_{\varphi}-<\mu^{\circ \circ} N^{\circ \circ} \overrightarrow{\boldsymbol{u}}^{\circ \circ}>_{\varphi}
\end{aligned}
$$

The part $<N^{\circ}>_{\varphi}\left(<\overrightarrow{\boldsymbol{v}}^{\circ}>_{\varphi}-<\overrightarrow{\boldsymbol{u}}^{\circ}>_{\varphi}\right)$ of (17) represents dispersion of the dust particles due to fluctuations in the average relative velocity vector. The following cases arise.

a) If the particle distribution is uniform, then $<N^{\circ}>_{\varphi}=0$, which indicates the absence of dispersion of dust particles.

b) The leading term on the right-hand-side of (17) involves a product of three averaged deviations. Due to the smoothing action of intrinsic volume averaging, it can be argued that each of the deviations in velocity, viscosity and number density is small enough that results in a negligible product of deviations.

c) If $\left\langle\overrightarrow{\boldsymbol{u}}^{\circ}\right\rangle_{\varphi}$ and $\left\langle\overrightarrow{\boldsymbol{v}}^{0}\right\rangle_{\varphi}$ are of similar magnitudes, their difference is small and can be neglected. Hence, dispersion of dust particles is negligible.

d) In cases where there is significant dispersion due to fluctuations in the average relative velocity, and viscosity deviations are significant, hydrodynamic dispersion may be modelled as a diffusion process involving a product of a diffusion coefficient vector, $\vec{\varepsilon}$, and a number density driving differential, $<$ $N>_{\varphi}-N_{d}$, where $N_{d}$ is an average reference particle distribution, $[13,14]$. We can thus write

$<\mu^{\circ}>_{\varphi}<N^{\circ}>_{\varphi}\left(<\overrightarrow{\boldsymbol{u}}^{\circ}>_{\varphi}-<\overrightarrow{\boldsymbol{v}}^{\circ}>_{\varphi}\right)=\vec{\varepsilon}(<$

$\left.N>{ }_{\varphi}-N_{d}\right)$

The term $\boldsymbol{\nabla} \cdot \varphi<N^{\circ} \overrightarrow{\boldsymbol{v}}^{\circ}>_{\varphi}$ appearing in the dustphase continuity equation (13) can be written as

$$
\begin{aligned}
& \boldsymbol{\nabla} \cdot \varphi<N^{\circ} \overrightarrow{\boldsymbol{v}}^{\circ}>_{\varphi}=\boldsymbol{\nabla} \cdot \varphi<N^{\circ}>_{\varphi}<\overrightarrow{\boldsymbol{v}}^{\circ}>_{\varphi}+\boldsymbol{\nabla} . \\
& \varphi<N^{\circ 0} \overrightarrow{\boldsymbol{v}}^{\circ}>_{\varphi}
\end{aligned}
$$

Three cases arise regarding (19):

a) If the particle distribution is uniform, then $<$ $N^{\circ}>_{\varphi}=0$, and the term $\boldsymbol{\nabla} \cdot \varphi<N^{\circ} \overrightarrow{\boldsymbol{v}}^{\circ}>_{\varphi}$ vanishes.

b) If the particle distribution is non-uniform but the fluctuations in dust-phase velocity and number density are small due to the absence of high velocity and porosity gradients, then the product of deviations in (19) is negligible.

c) Dust-phase continuity equation (3) has a right-hand-side of zero, indicating the absence of sources and sinks. One interpretation is that in free-space particle capture mechanisms and particle settling are ignored. When a porous matrix is introduced, the surface area within the REV is significantly high, and it is quite possible that capture mechanisms and particle settling become significant. It is then justifiable to model dust-particle mass transfer as a function of the total surface area, $S$, of the solid matrix that is in contact with the fluid, and express the term $\boldsymbol{\nabla} \cdot \varphi<N^{\circ}>_{\varphi}<$ $\overrightarrow{\boldsymbol{v}}^{\circ}>_{\varphi}$ as $\sigma S$, where $\sigma$ is a dust particle mass transfer coefficient. The surface area, $S$, depends on the porous microstructure. For example, Du Plessis and Masliyah [18] provided the following expression for $S$ when the porous sediment is granular:

$$
S=6 l^{2}(1-\varphi)^{2 / 3}
$$

where $l$ is a microscopic length scale of the granular representative unit cell (RUC), defined to be the 
smallest REV in which the porous medium quantities are embedded.

The term $\left\langle N^{\circ}\left(\nabla p_{d}\right)^{\circ}\right\rangle_{\varphi}$ in equation (14) vanishes if the particle distribution is uniform. If the particle distribution is non-uniform, this term vanishes as well in light of the fact that there will not be a dust-phase partial pressure.

The term $\boldsymbol{\nabla} \cdot \varphi<\overrightarrow{\boldsymbol{T}}>_{\varphi}$ appearing in the averaged fluid-phase momentum equation can be written as

$\boldsymbol{\nabla} \cdot \varphi<\mu \overrightarrow{\boldsymbol{I}}>_{\varphi}$, where $\overrightarrow{\boldsymbol{I}}=\left(\boldsymbol{\nabla} \overrightarrow{\boldsymbol{u}}+\boldsymbol{\nabla} \overrightarrow{\boldsymbol{u}}^{\boldsymbol{T}}\right)$, and can be expanded into the form

$\boldsymbol{\nabla} \cdot \varphi<\mu \overrightarrow{\boldsymbol{I}}>_{\varphi}=\boldsymbol{\nabla} \cdot \varphi<\mu>_{\varphi}<\overrightarrow{\boldsymbol{I}}>_{\varphi}+\boldsymbol{\nabla} \cdot \varphi(<$

$\left.\mu^{\circ}>_{\varphi}<\overrightarrow{\boldsymbol{I}}^{\circ}>_{\varphi}+<\mu^{\circ \circ} \overrightarrow{\boldsymbol{I}}^{\circ \circ}>_{\varphi}\right)$

The last term on the right-hand-side of (21) involves products of deviations of the average fluidphase velocity gradients and deviations from the average viscosity, which are small (hence ignored), in the absence of high fluid velocity and viscosity gradients. Equation (21) thus takes the form:

$$
\boldsymbol{\nabla} \cdot \varphi<\overrightarrow{\boldsymbol{T}}>_{\varphi}=\boldsymbol{\nabla} \cdot \varphi<\mu>_{\varphi}<\overrightarrow{\boldsymbol{I}}>_{\varphi}
$$

\subsection{Analysis of the Surface Integrals}

The surface integral appearing in continuity equation (11) can be evaluated by invoking Gauss' Divergence Theorem, and writing the surface integrals as:

$\int_{S} \overrightarrow{\boldsymbol{u}} \cdot \overrightarrow{\boldsymbol{n}} d S=\int_{V_{\varphi}} \boldsymbol{\nabla} \cdot \overrightarrow{\boldsymbol{u}} d V$

Making use of continuity equations (1), integral (23) vanishes.

The surface integrals appearing in continuity equation (13) is also evaluated by invoking Gauss' Divergence Theorem, and writing the surface integrals as:

$$
\int_{S} N \overrightarrow{\boldsymbol{v}} \cdot \overrightarrow{\boldsymbol{n}} d S=\int_{V_{\varphi}} \boldsymbol{\nabla} \cdot N \overrightarrow{\boldsymbol{v}} d V
$$

Making use of continuity equation (3), integral (24) vanishes.

The surface integrals $\int_{S} \overrightarrow{\boldsymbol{u}} \overrightarrow{\boldsymbol{u}} \cdot \overrightarrow{\boldsymbol{n}} d S$ and $\int_{S} N \overrightarrow{\boldsymbol{v}} \overrightarrow{\boldsymbol{v}}$. $\overrightarrow{\boldsymbol{n}} d S$ that appear in the fluid- and dust-phase momentum equations (12) and (14), respectively, are representative of shear forces. In the absence of a dust-phase viscosity, particle shear is zero and the surface integral $\int_{S} N \overrightarrow{\boldsymbol{v}} \overrightarrow{\boldsymbol{v}} \cdot \overrightarrow{\boldsymbol{n}} d S$ vanishes. In addition, the vanishing of normal component of fluid velocity on solid boundary translates into a no-slip condition on the solid matrix. According to rule (x), the surface integral $\int_{S} \overrightarrow{\boldsymbol{u}} \overrightarrow{\boldsymbol{u}} \cdot \overrightarrow{\boldsymbol{n}} d S$ vanishes.

Now, the surface integral appearing in equation (14) represents the total surface force due to the dustparticle distribution. It is composed of a pressure component, namely $-\frac{N}{V} \int_{S} p_{d}{ }^{\circ} \overrightarrow{\boldsymbol{n}} d S$, and a shear component, namely $\frac{m}{V} \int_{S} N \overrightarrow{\boldsymbol{v}} \overrightarrow{\boldsymbol{v}} \cdot \overrightarrow{\boldsymbol{n}} d S$.

The absence of particle shear renders the shear component insignificant, as we have already discussed above. However, the pressure component is the dust-phase partial pressure net surface force that is of significance unless the dust-phase inertia is negligible (as in the case of slow flow) or unless the particles represent a small bulk fraction by volume (as in the case of Saffman's dusty gas model). Accordingly, this net surface pressure force is ignored in this work.

The surface integrals, $\frac{1}{V} \int_{S} p^{\circ} \overrightarrow{\boldsymbol{n}} d S$ and $\frac{1}{V} \int_{S} \overrightarrow{\boldsymbol{T}}$. $\overrightarrow{\boldsymbol{n}} d S$ that appear in the fluid-phase momentum equations (10), can be combined into the surface filter $\left.\frac{1}{V} \int_{S} \overrightarrow{(\boldsymbol{T}} \cdot \overrightarrow{\boldsymbol{n}}-p^{\circ} \overrightarrow{\boldsymbol{n}}\right) d S$. This form of surface integral has been abundantly analyzed in the literature for both single-phase and dusty gas flows in porous media, $[7,8,17,18]$. Since the solid porous matrix affects the fluid-particle mixture through the portion of the surface area of the solid that is in contact with it, this surface integral contains the information necessary to quantify the forces exerted on the flowing fluid by the porous matrix. These forces give rise to Darcy resistance and the Forchheimer inertial and drag effects.

The above surface integral contains gradients of fluid-phase velocities, fluid pressure and viscosity. It is customary, however, to express the Darcy resistance and the Forchheimer term as $-\frac{\langle\mu\rangle_{\varphi}}{\eta} \varphi<$ $\overrightarrow{\boldsymbol{u}}>_{\varphi} \quad$ and $\quad-\frac{\rho C_{d}}{\sqrt{\eta}} \varphi<\overrightarrow{\boldsymbol{u}}>_{\varphi}\left|\varphi<\overrightarrow{\boldsymbol{u}}>_{\varphi}\right|$, respectively, where $C_{d}$ is the Forchheimer drag coefficient.

\subsection{Final Forms of Averaged Equations}


Taking into account the evaluations and approximations of surface integrals and deviation terms in the averaged fluid- and dust-phase equations (11)-(14), the governing equations are reduced to the following.

\section{For fluid-phase:}

\section{Continuity Equation:}

Equation (11) reduces to:

$\boldsymbol{\nabla} \cdot \varphi<\overrightarrow{\boldsymbol{u}}>_{\varphi}=0$

\section{Momentum Equations:}

Equation (12) reduces to:

$\rho \boldsymbol{\nabla} \cdot \varphi<\overrightarrow{\boldsymbol{u}}>_{\varphi}<\overrightarrow{\boldsymbol{u}}>_{\varphi}=-\varphi \nabla<p>_{\varphi}+\boldsymbol{\nabla} \cdot \varphi<$ $\overrightarrow{\boldsymbol{T}}>_{\varphi}+\frac{9}{2 a^{2}} \varphi<\mu>_{\varphi}<N>_{\varphi}\left[<\overrightarrow{\boldsymbol{v}}>_{\varphi}-\overrightarrow{\boldsymbol{u}}>_{\varphi}\right]-$ $\frac{9}{2 a^{2}} \varphi \vec{\varepsilon}\left(<N>_{\varphi}-N_{d}\right)-\frac{<\mu>_{\varphi}}{\eta} \varphi<\overrightarrow{\boldsymbol{u}}>_{\varphi}-\frac{\rho C_{d}}{\sqrt{\eta}} \varphi<$ $\overrightarrow{\boldsymbol{u}}>_{\varphi}\left|\varphi<\overrightarrow{\boldsymbol{u}}>_{\varphi}\right|$

\section{For dust-phase:}

\section{Continuity Equation:}

$\boldsymbol{\nabla} \cdot \varphi<N>_{\varphi}<\overrightarrow{\boldsymbol{v}}>_{\varphi}=-\sigma S$

\section{Momentum Equations:}

Equation (14) reduces to:

$m \boldsymbol{\nabla} \cdot \varphi<N>_{\varphi}<\overrightarrow{\boldsymbol{v}}>_{\varphi}<\overrightarrow{\boldsymbol{v}}>_{\varphi}=-\varphi<N>_{\varphi} \nabla<$ $p_{d}>_{\varphi}+\frac{9}{2 a^{2}} \varphi<\mu>_{\varphi}<N>_{\varphi}\left(<\overrightarrow{\boldsymbol{u}}>_{\varphi}-<\right.$

$\left.\overrightarrow{\boldsymbol{v}}>_{\varphi}\right)+\frac{9}{2 a^{2}} \varphi \vec{\varepsilon}\left(<N>_{\varphi}-N_{d}\right)$

Letting $\overrightarrow{\boldsymbol{V}}=<\overrightarrow{\boldsymbol{v}}>_{\varphi}, \overrightarrow{\boldsymbol{U}}=<\overrightarrow{\boldsymbol{u}}>_{\varphi}, \mu^{*}=<\mu>_{\varphi}$, $N^{*}=<N>_{\varphi}, p^{*}=<p>_{\varphi}, p_{d}^{*}=<p_{d}>_{\varphi}$, and

$\overrightarrow{\boldsymbol{I}}^{*}=<\overrightarrow{\boldsymbol{I}}>_{\varphi}=\left(\boldsymbol{\nabla} \overrightarrow{\boldsymbol{U}}+\boldsymbol{\nabla} \overrightarrow{\boldsymbol{U}}^{\boldsymbol{T}}\right), \quad$ equations $\quad(25)-(28)$ can be written in the following equivalent forms, respectively

$\boldsymbol{\nabla} \cdot \varphi \overrightarrow{\boldsymbol{U}}=0$

$\rho \boldsymbol{\nabla} \cdot \varphi \overrightarrow{\boldsymbol{U} \boldsymbol{U}}=-\varphi \nabla p^{*}+\boldsymbol{\nabla} \cdot \varphi \mu^{*} \overrightarrow{\boldsymbol{I}}^{*}$

$+\frac{9}{2 a^{2}} \varphi \mu^{*} N^{*}[\overrightarrow{\boldsymbol{V}}-\overrightarrow{\boldsymbol{U}}]-\frac{9}{2 a^{2}} \varphi \vec{\varepsilon}\left(N^{*}-N_{d}\right)-$

$\frac{\mu^{*}}{\eta} \varphi \overrightarrow{\boldsymbol{U}}-\frac{\rho C_{d}}{\sqrt{\eta}} \varphi^{2} \overrightarrow{\boldsymbol{U}}|\overrightarrow{\boldsymbol{U}}|$ $\boldsymbol{\nabla} \cdot \varphi N^{*} \overrightarrow{\boldsymbol{V}}=-\sigma S$

$m \boldsymbol{\nabla} \cdot \varphi N^{*} \overrightarrow{\boldsymbol{V}} \overrightarrow{\boldsymbol{V}}=-\varphi N^{*} \nabla p_{d}^{*}+\frac{9}{2 a^{2}} \varphi \mu^{*} N^{*}(\overrightarrow{\boldsymbol{U}}-\overrightarrow{\boldsymbol{V}})+$ $\frac{9}{2 a^{2}} \varphi \vec{\varepsilon}\left(N^{*}-N_{d}\right)$

If porosity $\varphi$ is constant, we can factor it out in equations (29)-(31).

If the particle distribution $N^{*}$ is non-uniform, then $p_{d}^{*}=0$ in equation (32).

If the particle distribution $N^{*}$ is uniform, then equations (29)-(32) take the following forms when porosity is constant and $C_{d}^{*}=\varphi C_{d}$ :

$\boldsymbol{\nabla} \cdot \overrightarrow{\boldsymbol{U}}=0$

$\rho \boldsymbol{\nabla} \cdot \overrightarrow{\boldsymbol{U}} \overrightarrow{\boldsymbol{U}}=-\nabla p^{*}+\boldsymbol{\nabla} \cdot \mu^{*} \overrightarrow{\boldsymbol{I}}^{*}+\frac{9}{2 a^{2}} \mu^{*} N^{*}[\overrightarrow{\boldsymbol{V}}-\overrightarrow{\boldsymbol{U}}]-$

$\frac{\mu^{*}}{\eta} \overrightarrow{\boldsymbol{U}}-\frac{\rho c_{d}^{*}}{\sqrt{\eta}} \overrightarrow{\boldsymbol{U}}|\overrightarrow{\boldsymbol{U}}|$

$\boldsymbol{\nabla} \cdot \overrightarrow{\boldsymbol{V}}=0$

$m \boldsymbol{\nabla} \cdot \overrightarrow{\boldsymbol{V}} \overrightarrow{\boldsymbol{V}}=-\nabla p_{d}^{*}+\frac{9}{2 a^{2}} \mu^{*}(\overrightarrow{\boldsymbol{U}}-\overrightarrow{\boldsymbol{V}})$

Equations (29)-(32) represent an underdeterminate system of eight scalar equations in the nine unknowns $\overrightarrow{\boldsymbol{U}}, \overrightarrow{\boldsymbol{V}}, p^{*}, p_{d}^{*}$ and $\mu^{*}$. In order to render this system determinate, we assume that viscosity is a known function of fluid pressure, $\mu^{*}=\mu^{*}\left(p^{*}\right)$. Many such functions are available in the literature, with a popular choice being Barus' equation, [15, $16]$, given by:

$\mu(p)=\mu_{0} e^{\alpha\left(p-p_{0}\right)}$

where $\alpha>0, p_{0}$ is a given reference pressure, and $\mu_{0}=\mu\left(p_{0}\right)$ is the fluid viscosity at the reference pressure. For small $\alpha$, or small pressure differences, (33) is approximated by

$\mu(p)=\mu_{0}\left[1+\alpha\left(p-p_{0}\right)\right]$.

\section{Conclusion}

In this work, we used the method of intrinsic volume averaging to develop model equations governing the flow of a variable viscosity fluid-particle mixture through a porous structure. Modelling flexibility was offered in the model by introducing a particle-phase partial pressure, interpreted as the pressure needed to maintain a uniform particle number density. 
Variations in viscosity are assumed to be due to pressure variations, and Stokes' coefficient of resistance is expressed in a form that includes local viscosity variations. Effects of the porous microstructure on the flowing mixture are manifested through the Darcy resistance and the Forchheimer inertial term.

\section{References:}

[1] Fusi, L., Farina, A. and Rosso, F., Mathematical Models for Fluids with Pressure Dependent Viscosity Flowing in Porous Media. International Journal of Engineering Science, Vol. 87, 2015, pp. 110-118.

[2] Housiadas, K.D., Georgiou, G.C. and Tanner, R.I., A Note on the Unbounded Creeping Flow Past a Sphere for Newtonian Fluids with PressureDependent Viscosity. Int. Journal of Engineering Science, Vol. 86, 2015, pp. 1-9.

[3] Nakshatrala, K.B. and Rajagopal, K.R. (2011). A Numerical Study of Fluids with Pressure-Dependent Viscosity Flowing through a Rigid Porous Medium. Int. J. Numer. Meth. Fluids, Vol. 67, 2011, pp. 342368.

[4] Chang, J., Nakashatrala, K.B. and Reddy, J.N., Modification to Darcy-Forchheimer Model Due to Pressure-Dependent Viscosity: Consequences and Numerical Solutions. J. Porous Media, Vol. 20, No. 3, 2017, pp. 263-285.

[5] Abu Zaytoon, M.S., Allan, F.M., Alderson, T.L. and Hamdan, M.H., Averaged Equations of Flow of Fluid with Pressure-Dependent Viscosity through Porous Media. Elixir Appl. Math., Vol. 96, 2016, pp. 41336-41340.

[6] Alharbi, S.O., Alderson, T.L. and Hamdan, M.H., Flow of a Fluid with Pressure-Dependent Viscosity through Porous Media", Advances in Theoretical and Applied Mechanics, vol. 9(1), 2016, pp. 1-9.

[7] Abu Zaytoon, M.S. and Hamdan, M.H., The Flow of a Saffman's Dusty Gas with Pressure-Dependent Viscosity through Porous Media Elixir Appl. Math., Vol. 98, 2016, pp. 42550-42554

[8] Roach, D.C., Abu Zaytoon, M.S. and Hamdan, M.H., On the Flow of Dusty Gases with Pressure Dependent Viscosities through Porous Structures, Int. J. Enhanced Research in Science, Technology \& Engineering, Vol. 5, No. 9, 2016, pp. 46-54.
[9] Saffman, P.G., On the Stability of Laminar Flow of a Dusty Gas, J. Fluid Mechanics, Vol. 13, No. 1, 1962, pp. 120-128.

[10] Marble, F. E., Dynamics of Dusty Gases, Annual Review of Fluid Mechanics, Vol. 2, 1970, pp. 397-446.

[11] Hamdan, M.H. and Barron, R.M., On the DarcyLapwood-Brinkman-Saffman Dusty Fluid Flow Models in Porous Media. Part I: Models Development, Applied Mathematics and Computation, 54, No. 1, 1993, pp. 65-79.

[12] Hamdan, M.H. and Barron, R.M., On the DarcyLapwood-Brinkman-Saffman Dusty Fluid Flow Models in Porous Media. Part II: Applications to Flow into a Two-Dimensional Sink, Applied Mathematics and Computation, 54, No.1, 1993, pp. 81-97.

[13] Alzahrani, S.M. and Hamdan, M.H., GasParticulate Models of Flow through Porous Structures, Int. J. Engineering Research and Applications, Vol. 6, No.2(3), 2016, pp.54-59.

[14] Alzahrani, S.M. and Hamdan, M.H., Mathematical Modelling of Dusty Gas Flow through Isotropic Porous Media with Forchheimer Effects, Int. J. of Enhanced Research in Science, Technology \& Engineering, Vol. 5, No. 5, 2016, pp. 116-124.

[15] Barus, C.J., Note on Dependence of Viscosity on Pressure and Temperature. Proceedings of the American Academy, Vol. 27, 1891, pp. 13-19.

[16] Barus, C.J., Isothermals, Isopiestics and Isometrics Relative to Viscosity. American Journal of Science, Vol. 45, 1893, pp. 87-96.

[17] Du Plessis, J.P. and Masliyah, J.H., Mathematical Modeling of Flow through Consolidated Isotropic Porous Media, Transport in Porous Media, Vol. 3, 1988, pp. 145-161.

[18] Du Plessis, J.P. and Masliyah, J.H., Flow through Isotropic Granular Porous Media, Transport in Porous Media, Vol. 6, 1991, pp. 207-221.

\section{Contribution of individual authors to the creation of a scientific article (ghostwriting policy)}

All three authors participated in pertinent literature review to identify missing state-of-the-art knowledge 
in this area of research. All three authors independently obtained the averaged governing equations.

S. Jayyousi Dajani outlined the steps to take in conducting this research.

M.S. Abu Zaytoon double checked the equations for consistency with the literature.

M.H. Hamdan collected information from co-authors and wrote the manuscript.

\section{Sources of funding for research presented in a scientific article or scientific article itself}

No sources of funding for this work. Support from authors' institutions in the form of office space to work in and internet connections.

\section{Creative Commons Attribution}

\section{License 4.0 (Attribution 4.0 International, CC BY 4.0)}

This article is published under the terms of the Creative Commons Attribution License 4.0 https://creativecommons.org/licenses/by/4.0/deed.en US 